\title{
Mortality associated with lithium and valproate treatment of US Veterans Health Administration patients with mental disorders
}

Eric G. Smith, Karen L. Austin, Hyungjin Myra Kim, Susan V. Eisen, Amy M. Kilbourne, Donald R. Miller, Kara Zivin, Claire Hannemann, Brian C. Sauer and Marcia Valenstein

\section{Background}

The mood stabilisers lithium and valproate might plausibly have differing associations with mortality because of differing effects on mental health and various physiological indicators.

\section{Aims}

To assess associations between lithium, valproate and nonsuicide mortality.

\section{Method}

Intention-to-treat, propensity score-matched cohort study.

\section{Results}

Lithium was associated with significantly reduced non-suicide mortality in the intent-to-treat cohort over 0-90 days (hazard ratio $(H R)=0.67,95 \% \mathrm{Cl} 0.51-0.87$ ) but not longer. In secondary analyses, a sizeable reduction in mortality was observed during active treatment with lithium across all time periods studied (for example 365-day HR $=0.62$ $95 \% \mathrm{Cl} 0.45-0.84)$, but significantly increased risks were observed among patients discontinuing lithium by 180 days $(\mathrm{HR}=1.54,95 \% \mathrm{Cl} 1.01-2.37)$

\section{Conclusions}

Patients initiating lithium had lower non-suicide mortality over 0-90 days than patients initiating valproate and consistently lower non-suicide mortality among patients maintaining treatment, but elevated risk among patients discontinuing treatment by 180 days. Although residual confounding or selection effects cannot be excluded, this study suggests potential benefits to enhancing lithium treatment persistence and the monitoring of patients discontinuing lithium. There is a need for further research.

\section{Declaration of interest}

None.

\section{Copyright and usage}

(c) The Royal College of Psychiatrists 2015.
Although a substantial literature exists concerning the potential association of lithium with reduced suicide mortality, very few studies have examined lithium's potential influence on non-suicide mortality. Many organ systems are exposed to lithium, ${ }^{1,2}$ and lithium produces diverse physiological effects. Some of these effects are potentially beneficial (for example leukocytosis, ${ }^{3}$ reduced heart rate ${ }^{4}$ and neurogenesis ${ }^{5}$ ), whereas others are potentially hazardous (for example renal ${ }^{6,7}$ and thyroid insufficiency, ${ }^{8}$ QTc prolongation ${ }^{4}$ or arrhythmias $\left.{ }^{9-11}\right)$. A limited number of studies, primarily ${ }^{12-19}$ but not exclusively ${ }^{20}$ non-randomised, have examined associations between lithium treatment and non-suicide mortality. These studies are consistent with the possibility that lithium might reduce mortality risk in psychiatric patients. Determining the effects on non-suicide mortality of lithium or other psychiatric treatments is clearly important, especially since patients with serious mental illness are at particular risk for premature mortality. ${ }^{21-24}$

We conducted a nationwide cohort study of the US Veterans Health Administration's (VHA) detailed clinical databases, employing two methods intended to increase the likelihood that observational studies will yield results similar to randomised trials: high-dimensional propensity score (hdPS) matching and intent-to-treat estimates. The hdPS matching permit inclusion of particularly detailed information concerning potential confounding while facilitating the assessment of the balance in these potential confounders that is achieved between treatment groups. Intent-to-treat estimates enhance interpretation of results by allowing assessment of whether benefits during active treatment are negated by risks upon discontinuation. Employing these approaches, we investigated whether initiation of lithium was associated with reduced non-suicide mortality compared with initiation of valproate, a treatment that has largely replaced lithium in many countries. ${ }^{25-28}$

\section{Method}

\section{Data sources}

Demographic characteristics, in-patient and out-patient mental and non-mental health treatment records, and out-patient pharmacy prescription data were obtained from the VHA National Psychosis and Depression Registries. ${ }^{29}$ (These registries are linked, de-identified healthcare databases of all VHA patients nationwide since 1997 with at least one psychotic or depressive disorder diagnosis). This study was approved by the Institutional Review Boards of the Bedford and Ann Arbor Veterans Affairs Medical Centers.

\section{Study cohort}

Incident users ${ }^{30}$ ( $\geqslant 6$ months of no lithium or valproate use but with recent VHA utilisation) receiving at least one out-patient prescription for lithium or valproate from April 1999 to December 2008 were identified (online Fig. DS1). A broad cohort of patients with mood or psychotic diagnoses in the 30 days prior to medication initiation was examined since the limited prior literature concerning lithium and mortality is not restricted to bipolar disorder (online supplement DS1). ${ }^{13,15,19,20}$

Patients with possible non-psychiatric indications for valproate or lithium (epilepsy, migraine or cluster headache, or neuropathy diagnoses in the past 30 days; dementia medication use in the past 180 days; cancer, dementia, skull fracture diagnosis, traumatic brain injury diagnosis or treatment, home care, or hospice care in the past year; or any nursing home residence or 
in-patient rehabilitation in the past 2 years) were excluded. Patients were also excluded if they initiated lithium or valproate on an 'as needed' basis or both medications simultaneously, or resided outside the USA.

\section{Exposure determination}

Receipt of lithium or valproate was defined by a patient filling an out-patient prescription for these medications. For the intent-to-treat analysis, all patients filling an initial out-patient prescription were followed until the end of the follow-up (i.e. 90,180 or 365 days) or death. Secondary analyses stratified follow-up time by whether patients were still receiving initial treatment. Patients were considered 'as-initially treated' until a $\geqslant 15$-day gap occurred between out-patient prescriptions (adjusting for early refills) or upon initiation of the other mood stabiliser (i.e. lithium or valproate). 'Former users' consisted of patients after initial treatment discontinuation until follow-up time was censored upon treatment resumption, treatment switching (for example patients receiving valproate as initial treatment who switch to lithium), death or the end of follow-up. We will use the term 'former users' to designate patients within the period of time during which they have stopped their initial treatment and were not exposed to either lithium or valproate. ('Former user' exposure time was censored if the patient resumed either medication.) Given that this 'former user' follow-up period is free from exposure to either medication studied, former users have been advanced as a potential index of residual baseline confounding and/or selection occurring during treatment. ${ }^{31,32}$ However, risks among former users can be more fully conceptualised as representing the sum total of effects of residual confounding and selection along with any persistence of effects from active treatment, and any risks produced upon treatment discontinuation (online supplement DS2). This last category of 'discontinuationassociated risks' would include effects such as rebound mania or depression.

\section{Outcome}

Date and cause of death was obtained from National Death Index files for 1999-2009. ${ }^{33}$ This study was limited to non-suicide mortality, with follow-up time for patients dying of suicide censored at suicide death.

\section{Propensity score modelling}

In total, 948 covariates derived from VHA databases were included in an initial propensity score model generally following the hdPS approach $^{34,35}$ (online supplement DS3). These covariates included potential risk factors for both non-suicide and suicide mortality ${ }^{36}$ (including demographic characteristics, diagnoses, general VHA mental and non-mental health services utilisation, ${ }^{34}$ admissions to hospital, clinic use, occurrence of diagnostic testing, current and recent prescriptions, recent injuries and diagnosed suicide attempts, and state-level and VHA-hospital subsystem mortality risk $^{37}$ ) (online Table DS1 and online supplement DS4), often with multiple indicator variables to allow for non-linear covariatemortality relationships. An 'outcome-focused' propensity score was then derived limiting covariates to the 523 covariates with substantial associations with outcome ${ }^{38}$ (i.e. $\pm 20 \%$ change in non-suicide mortality). ${ }^{39}$ Further details of how this covariate restriction was implemented are provided in online supplement DS4. This outcome-focused propensity score, intended to limit unintended amplification of confounding that remained uncontrolled, ${ }^{38,40,41}$ provided the basis for the results reported here. Results from analyses using the initial propensity score are provided in online supplement DS5. The results from the two models are generally similar but differ in a few important details, such as the time periods for which significant associations are detected.

\section{Statistical methods}

The propensity score was calculated using logistic regression. Patients initiating lithium and valproate were 1:1-matched (online supplement DS6) using callipers of 0.2 standard deviations of the propensity score logit, ${ }^{42,43}$ resulting in $99.3 \%$ matching of lithium-initiated patients. Balance in covariates between treatment groups was assessed using standardised differences (equivalent to Cohen's $d$ effect sizes, with a difference of $>0.10$ indicating significant imbalance). ${ }^{44}$

Statistical significance was determined using techniques that reflected matching (stratified Cox regression with sandwich variance estimators) for the primary intent-to-treat analyses and the secondary as-treated analyses. Ordinary Cox regression was used for the secondary former user analysis (since matching was not preserved for this analysis). All analyses except standardised differences were performed using SAS, version 9.3. Standardised differences were calculated using Microsoft Excel 2010.

\section{Results}

The incident user cohort of 93162 patients initiating lithium or valproate was generally balanced (i.e. standardised differences between the treatment groups $<0.10)^{44}$ in virtually all non-mental health and mental health covariates studied, even prior to matching. After matching, substantial additional balance was achieved between treatment groups ( $n=21288$ patients per group). As an end result, hdPS matching achieved a very close balance (standardised differences $<0.019$ ) for each one of the 523 covariates included in the outcome-focused analysis. Table 1 and 2 indicate the standardised differences in the matched cohort for two categories of covariates: (a) those few variables with a substantial imbalance ( $\geqslant 0.10$ standardised difference) between treatment groups initially ( 8 of 523 covariates, or $1.5 \%$, Table 1), and (b) a number of additional covariates with well-established or highly plausible relationships with non-suicide mortality (Table 2). These additional covariates include age, disability status, recent number and types of hospital admissions, diagnoses, medications, and attendance at certain out-patient clinics. Comparison of the unmatched and hdPS-matched treatment effect estimates indicate that hdPS-matching reduced effect sizes in a direction consistent with reducing baseline confounding biasing against valproate (i.e. reducing differences that placed patients initiating valproate at higher intrinsic risk of non-suicide mortality) (online supplement DS7).

It should be noted that the gender ratio of our sample (Table 1) differs substantially from that found for bipolar disorder in the general population because our sample is a Veteran sample, primarily made up of men. It is particularly important to note that the hdPS-matching procedure did not substantially change the prevalence of male or female gender in the final study cohort compared with the initial sample, or the prevalence of any of the other included covariates. Rather, the matching procedure led to the selection of a set of patients initiating valproate who were very similar to those initiating lithium. For instance, in the original study cohort prior to matching $13.9 \%$ of patients initiating lithium and $9.4 \%$ of patients initiating valproate were women. In the final cohort, $13.8 \%$ of patients initiating lithium and $13.9 \%$ of patients initiating valproate were women.

Impersistence with treatment was very common even within 180 days, but rates of treatment impersistence were highly similar between the treatment groups: approximately $76.5 \%$ of patients 


\begin{tabular}{|c|c|c|c|}
\hline & Lithium, $n(\%)(n=21288)$ & Valproate $n(\%)(n=21288)$ & Standardised difference \\
\hline Gender, female ${ }^{a}$ & $2932(13.8)$ & $2952(13.9)$ & -0.003 \\
\hline Bipolar I disorder, past 30 days & $9630(45.2)$ & $9719(45.7)$ & -0.008 \\
\hline Other psychosis, past 30 days & $251(1.2)$ & $261(1.2)$ & -0.004 \\
\hline Post-traumatic stress disorder, past year & $4858(22.8)$ & $4849(22.8)$ & 0.001 \\
\hline Other mood stabiliser(s), current & $2963(13.9)$ & $2894(13.6)$ & 0.006 \\
\hline Prior mood stabiliser treatment & $7573(35.6)$ & 7473 (35.1) & 0.010 \\
\hline Mild liver disease, past year & $1795(8.4)$ & $1708(8.0)$ & 0.015 \\
\hline Angiotensin converting enzyme inhibitor, current & $2772(13.0)$ & $2765(13.0)$ & 0.001 \\
\hline
\end{tabular}

Table 2 Characteristics of patients initiating lithium and valproate for select additional variables with lesser initial imbalances (propensity-score matched sample)

Lithium, $n(\%)(n=21288) \quad$ Valproate $n(\%)(n=21288) \quad$ Standardised difference

\begin{tabular}{|c|c|c|c|}
\hline \multicolumn{4}{|l|}{ Age, years ${ }^{a}$} \\
\hline 65-79 & $1358(6.4)$ & 1359 (6.4) & 0.000 \\
\hline $80+$ & $164(0.8)$ & $154(0.7)$ & 0.005 \\
\hline Married & $7455(35.0)$ & $7370(34.6)$ & 0.008 \\
\hline Disability $(51-100 \%)$ & $5473(25.7)$ & $5481(25.7)$ & -0.001 \\
\hline Charlson Comorbidity Index, past year: $1+$ & 7601 (35.7) & $7468(35.1)$ & 0.013 \\
\hline Myocardial infarction, past year & $235(1.1)$ & $243(1.1)$ & -0.004 \\
\hline Diabetes (uncontrolled), past year & $2722(12.8)$ & $2691(12.6)$ & 0.004 \\
\hline Arrhythmia, past year & $860(4.0)$ & $856(4.0)$ & 0.001 \\
\hline Chronic obstructive pulmonary disease, past year & 2968 (13.9) & $2934(13.8)$ & 0.005 \\
\hline Total non-psychiatric medications, current: $5+$ & $5670(26.6)$ & $5589(26.3)$ & 0.009 \\
\hline Beta-blockers, current & $2757(13.0)$ & $2713(12.7)$ & 0.006 \\
\hline Opioid pain medication, current & 2409 (11.3) & $2374(11.2)$ & 0.005 \\
\hline Antiplatelet agent, current & $221(1.0)$ & $218(1.0)$ & 0.001 \\
\hline Warfarin, current & $200(0.9)$ & $187(0.9)$ & 0.006 \\
\hline Non-psychiatric admissions to hospital, past year: $1+$ & $1842(8.7)$ & $1781(8.4)$ & 0.010 \\
\hline Latest discharge from medical intensive care unit & $330(1.6)$ & $323(1.5)$ & 0.003 \\
\hline Latest discharge from neurology & $54(0.3)$ & $54(0.3)$ & 0.000 \\
\hline Non-psychiatric discharge, against medical advice, past year & $207(1.0)$ & $203(1.0)$ & 0.002 \\
\hline General surgery, past 180 days: $1+$ & 705 (3.3) & $697(3.3)$ & 0.002 \\
\hline Non-psychiatric visits, past 7 days: $1+$ & $4479(21.0)$ & $4426(20.8)$ & 0.006 \\
\hline Specialty visits, past 180 days: $1+$ & $3720(17.5)$ & $3619(17.0)$ & 0.013 \\
\hline Cardiology clinic, past 180 days: $1+$ & $594(2.8)$ & $599(2.8)$ & -0.001 \\
\hline Pain clinic, past 180 days: $1+$ & $447(2.1)$ & $467(2.2)$ & -0.006 \\
\hline Chaplain service, past 180 days: $1+$ & $547(2.6)$ & $540(2.5)$ & 0.002 \\
\hline Nuclear medicine, past year: $1+$ & $837(3.9)$ & $812(3.8)$ & 0.006 \\
\hline Alcohol dependence & $4440(20.9)$ & $4449(20.9)$ & -0.001 \\
\hline Heroin/opiate dependence & $824(3.9)$ & $810(3.8)$ & 0.003 \\
\hline
\end{tabular}

initiating lithium and $76.1 \%$ of patients initiating valproate did not persist with initial treatment for 180 days (online Table DS4).

Overall survival was greater among patients initiating lithium, with 274 deaths over 365 days observed among the lithium intent-to-treat cohort, compared with 296 deaths among the valproate intent-to-treat cohort. Greater differences (71 v. 101 deaths, respectively) were observed between the as-initially treated cohorts. Survival curves for the intent-to-treat and as-initially treated analyses are provided in Fig. 1.

Table 3 provides the primary, intent-to-treat analysis results, indicating that lithium was associated with substantially reduced mortality risks over $0-90$ days (hazard ratio $(\mathrm{HR})=0.67,95 \%$ CI $0.51-0.87$ ), the period of greatest medication persistence, but not $0-180$ days $(\mathrm{HR}=0.97,95 \%$ CI $0.82-1.15)$ or $0-365$ days $(\mathrm{HR}=0.92,95 \%$ CI $0.82-1.04)$.

Secondary analyses by treatment status (Table 4) reveal large and significant associations with non-suicide mortality during active lithium treatment compared with valproate treatment over all time periods. Hazard ratios were consistently and considerably lower during the period of likely active use of lithium compared with likely active use of valproate (as-initially treated hazard ratios ranging from $\mathrm{HR}=0.59,95 \%$ CI $0.42-0.82$ to $\mathrm{HR}=0.62$, $95 \%$ CI $0.45-0.84)$. However, significantly increased non-suicide mortality was also observed among lithium former users over $0-180$ days $(\mathrm{HR}=1.54,95 \%$ CI $1.01-2.37)$, although not other time periods. 


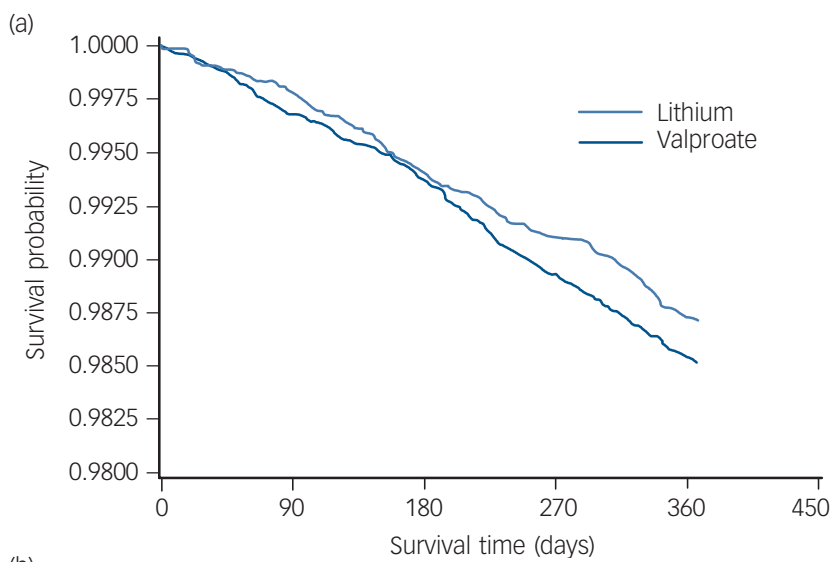

(b)

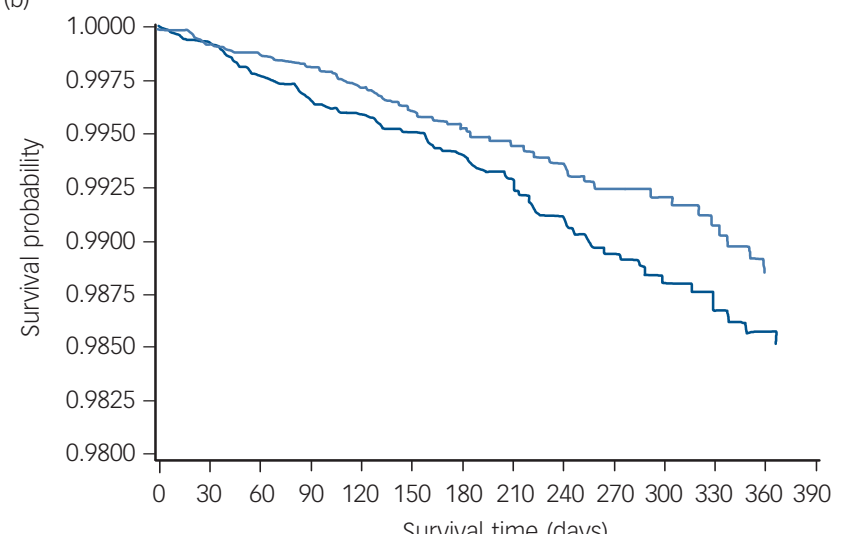

Fig. 1 Survival curve of lithium and valproate treatment for (a) intent-to-treat cohort and (b) as-treated patients.

Table 5 indicates no significant intent-to-treat associations existed between treatment and specific categories of causes of death at 365 days. Therefore, the observed hazard ratios, modestly above or below the null for each cause of death, possibly represent simple chance fluctuations. The mortality categories with associations the closest to statistical significance, were cardiovascular disease and deaths from all other causes. Upon further examination, these categories were also the only categories to have significant (all other causes, HR $=0.50,95 \%$ CI $0.28-0.91$ ) or borderline significant (cardiovascular disease, $\mathrm{HR}=0.60,95 \% \mathrm{CI}$ $0.36-1.01$ ) associations among as-initially treated individuals.

\section{Discussion}

\section{Main findings}

In a nationwide cohort study of 42576 VHA psychiatric patients initiating lithium and valproate, significant intent-to-treat associations of lithium initiation with lower mortality risk were observed over 0-90 days but not over 0-180 days or 0-365 days. In contrast, secondary analysis of patients who persisted with treatment, indicated strong associations with reduced mortality for lithium, compared with valproate, treatment across all time
Table 3 Risk of non-suicide mortality (intent-to-treat cohort) Time period, days Hazard ratio (lithium/valproate)

\begin{tabular}{|c|c|}
\hline $0-90$ & $0.67^{\mathrm{a}}(0.51-0.87)$ \\
\hline $0-180$ & $0.97^{b}(0.82-1.15)$ \\
\hline $0-365$ & $0.92^{\mathrm{C}}(0.82-1.04)$ \\
\hline \multicolumn{2}{|c|}{$\begin{array}{l}\text { a. Based on } 48 \text { deaths } / 1934388 \text { person-days for lithium and } 72 \text { deaths } / 1933337 \\
\text { person-days for valproate; } P=0.003 \text {. } \\
\text { b. Based on } 128 \text { deaths } / 3839959 \text { person-days for lithium and } 132 \text { deaths } / 3838384 \\
\text { person-days for valproate; } P=0.73 \text {. } \\
\text { C. Based on } 274 \text { deaths } / 733701 \text { person-days for lithium and } 296 \text { death } / 7729420 \\
\text { person-days for valproate; } P=0.17 \text {. }\end{array}$} \\
\hline
\end{tabular}

periods. However, the reduction in the intent-to-treat association from 0-90 to 0-180 days is very rapid, and our secondary analysis indicates significantly increased mortality risk associated with discontinuing lithium, compared with valproate, over 0-180 days of treatment. Both findings suggest a possible increase in mortality risk associated with lithium, compared with valproate, among patients who discontinue the medication (online supplement DS8). These findings, combined with the possibility that at least some level of confounding (most likely biasing towards worse outcomes in patients initiating valproate) may remain in our analyses, prevent firm conclusions concerning whether the initiation of lithium is associated with a net mortality benefit or harm. Regardless of this uncertainty, however, one clear and important clinical recommendation results from this mortality study: once lithium is initiated, persistence with lithium treatment should be monitored and, if clinically reasonable, maintained.

\section{Confounding}

Risks associated with lithium discontinuation could, in theory, potentially limit, eliminate, or even exceed mortality benefits associated from active lithium treatment when compared with valproate. Determining whether this occurs is difficult even though the intent-to-treat estimates significantly favour lithium treatment from 0-90 days and numerically, although not significantly, favour lithium treatment over longer time periods. Although intent-to-treat estimates are intended to reflect the balance of benefits and harms among all patients, regardless of whether continuing on or discontinuing treatment, even relatively small amounts of confounding biasing against valproate could alter risk-benefit judgements. Propensity score matching can achieve very close balance on measured factors (and did so here), however, factors that are either incompletely modelled or unmeasured in the analysis are intrinsically not able to be balanced by propensity score methods. Such factors might include provider tendencies to prescribe valproate to individuals who are more severely ill within the categories of illness that are balanced through the propensity score matching. Thus, not only does random error (as indicated by the lack of statistical significance) limit interpretation of the intent-to-treat hazard ratios after 90 days, but so does possible residual confounding (online supplements DS8 and DS9).

Although our hdPS successfully achieved close balance on a large variety of important potential confounders, some degree of

\begin{tabular}{|c|c|c|c|c|}
\hline \multirow[b]{2}{*}{ Time period } & \multicolumn{2}{|c|}{ During initial exposure (as-initially treated) } & \multicolumn{2}{|c|}{ During subsequent non-exposure (former users) } \\
\hline & Hazard ratio (lithium/valproate) & $P$ & Hazard ratio (lithium/valproate) & $P$ \\
\hline 0-90 days & $0.59(0.42-0.84)$ & 0.004 & $0.88(0.45-1.74)$ & NS \\
\hline 0-180 days & $0.59(0.42-0.82)$ & 0.002 & $1.54(1.01-2.36)$ & 0.045 \\
\hline 0-365 days & $0.62(0.45-0.84)$ & 0.002 & $1.02(0.79-1.32)$ & NS \\
\hline
\end{tabular}


Table 5 Risk of non-suicide mortality by cause (intent-to-treat cohort)

\begin{tabular}{|lcc|}
\hline Cause of death & Deaths, $n$ & $\begin{array}{c}\text { Intent-to-treat hazard } \\
\text { ratio (lithium/valproate) }\end{array}$ \\
\hline Cardiovascular & 171 & $0.86(0.70-1.06)$ \\
\hline Injury & 105 & $0.94(0.72-1.24)$ \\
\hline Cancer & 54 & $1.25(0.85-1.83)$ \\
\hline Stroke & 21 & $1.20(0.66-2.18)$ \\
\hline All other causes & 231 & $0.87(0.71-1.05)$ \\
\hline
\end{tabular}

remaining confounding is plausible. Risk estimates indicating greater mortality risk among patients initiating valproate in the unmatched sample (online supplement DS7) become less pronounced after hdPS-matching, suggesting overall confounding initially biases against valproate. Also, the former-user hazard ratio for patients initiating, then stopping, lithium over 0-90 days compared with patients initiating, and then stopping, valproate, is $<1.0$ (although random error cannot be excluded). ('former users' are those patients no longer receiving either mood stabiliser, thus, this 'former user' period represents time not exposed to either lithium or valproate). This observation is consistent with the possibility that, if residual confounding does exist to at least some degree, then patients initiated on lithium were likely at lower baseline mortality risk on average than patients initiated on valproate. Finally, even though significantly increased mortality risk is observed among lithium former users from 0 to 180 days, the possibility of some confounding against valproate persisting throughout the analysis is suggested by the subsequent sharp decrease in former user risks from $0-180$ to $0-365$ days (central estimate $\mathrm{HR}=1.54$ changing to $\mathrm{HR}=1.02$ ) (online supplement DS8). Although, again, random error cannot be excluded, if any confounding does generally bias against valproate, this suggests that the mortality risk associated with patients who discontinued lithium over $0-180$ days compared with patients who discontinued valproate may be even greater than indicated.

Nevertheless, even if confounding biasing against valproate exists, the effect estimates for active lithium treatment compared with active valproate treatment are of such size (central estimate $\mathrm{HR}=0.59-0.62)$ as to command attention. Such as-initially treated estimates are often the only type of effect estimate typically provided in non-randomised treatment studies. In this study, these 'as-initially treated estimates may provide only part of the important information to consider, given the possibilities of confounding and discontinuation-associated risks. Nevertheless, even if some level of confounding persists after matching, it is unclear whether such confounding persists to such an extent to fully account for the significant intent-to-treat association observed early during follow-up, or as-initially treated associations observed throughout follow-up. This becomes a question for further research. Not focused upon here, but also a potential contributor to the secondary analysis results is differential selection of patients, once they have initiated treatment, to stop one medication (e.g. lithium) compared with the other medication (e.g. valproate). Such differential selection could occur from either providers or patients relying on different reasons when deciding to discontinue their initial treatment, depending on whether that initial treatment was lithium or valproate. This possibility is consistent with some, but not all, of the study findings (online supplement DS10).

\section{Importance of treatment persistence}

Regardless of these uncertainties, one clear clinical recommendation can be made: once initiated, persistence with lithium treatment should be monitored and, if clinically indicated, maintained.
Whether or not the predominant association of lithium treatment (compared with valproate) with mortality is one of lower mortality risks during active treatment or higher mortality risks during lithium discontinuation, in either case maximising persistence with lithium treatment would be of clear benefit. Treatment persistence would both minimise potential risks upon lithium discontinuation and maximise potential benefits resulting from active lithium treatment (online supplement DS11).

\section{Findings from other studies}

Our results are generally consistent with a limited prior literature. A clinical trial meta-analysis of both placebo and comparatorcontrolled trials reported significant reductions in overall mortality with lithium treatment $(\mathrm{HR}=0.42,95 \%$ CI $0.27-0.81)$; however, restriction to non-suicide mortality from active comparator trials results in the findings being informed by just 4 deaths among lithium recipients and 12 deaths among comparator recipients (see Cipriani et al Figures 2 and $4^{20}$ ). Non-randomised studies of lithium's effects on non-suicide mortality are few but generally indicate reduced risks with active lithium treatment, although they typically lack active comparators, intent-to-treat designs or detailed controls for potential confounding. ${ }^{12-19}$ Our study is, to our knowledge, the first non-randomised study to examine non-suicide mortality risks associated with lithium discontinuation in a study with an active comparator, but is consistent in a general sense with limited prior uncontrolled studies. These studies observed that lithium discontinuation is a high-risk period for overall mortality. ${ }^{45,46}$ Our study's findings potentially may also be broadly consistent with prior randomised ${ }^{47}$ and non-randomised ${ }^{48,49}$ literature indicating that lithium discontinuation substantially increases the risk of mood episodes. These risks for mood-episode recurrence were sufficiently pronounced that one author reviewing these studies even recommended that lithium not be initiated unless a patient was likely to complete 2 years of continuous treatment. ${ }^{50}$ Finally, our conclusions concerning the importance of persistence with lithium treatment are generally consistent with multiple prior studies reporting substantial lithium treatment impersistence. $^{51-57}$

\section{Future directions}

In our judgement, this study clearly establishes high-priority clinical and research agendas. The data from this study clearly suggests a need for clinical systems and providers to encourage patients to continue with their lithium treatment once it is initiated. A limited literature exists concerning psychosocial interventions that might help accomplish this task. ${ }^{58}$ In addition, two trials that have included group psychoeducation on the importance of medication treatment and/or adherence for successful management of bipolar disorder have shown superior outcomes to standard care. ${ }^{59,60}$ Our data also supports monitoring patients closely upon discontinuation when feasible, a practice already recommended in some guidelines to limit mood-episode recurrence. ${ }^{61}$ Finally, some approaches such as gradual discontinuation $^{49}$ have been proposed to limit the adverse psychiatric effects of lithium discontinuation.

From a research perspective, this study establishes a need for further non-randomised studies to elucidate the balance of risks and harms associated with lithium initiation. This might include evaluating cohorts with different demographics, treatment persistence rates, or psychiatric or non-psychiatric comorbidities. If additional methodological innovations could be combined with the approaches used here, further reductions in confounding or selection during treatment might result. For instance, if valid instrumental variables can be identified, these might reduce 
confounding from imperfectly measured or unmeasured factors. Marginal structural models might reduce the impact of differential selection during treatment, and allow the impact of additional treatments commenced during follow-up to be evaluated. Research consortiums have been recently developed to rapidly conduct research along such lines; ${ }^{62}$ our findings suggests that the mortality effects of lithium and comparators should be a high-priority target for these consortiums. Such non-randomised research could also help determine the need for subsequent randomised trials, although the acceptability of such trials is uncertain. The need for additional, rapidly initiated research arises from the obvious health relevance of even small differences in non-suicide mortality between these commonly used medications.

\section{Limitations}

Study limitations include our lack of in-patient prescription information, lack of serum medication levels as an alternative method to assess persistence with treatment and the inherent inability to completely model potentially important covariates such as admissions to hospital (online supplement DS12). A few variables found to be important in past mortality studies (income and ethnicity) that are sometimes poorly measured in Veterans Affairs data were not included in the outcome-focused propensity score. Although available medical information was extensively represented, this information was only present for treatment received at the VHA. Although we employed multiple methods to attempt to balance the treatment groups in VHA medical utilisation (including indicators such as the presence and number of recent non-psychiatric medications, overall visits and specialist visits a patient received), this lack of outside healthcare data may be particularly relevant for patients receiving emergency care (which is more likely to occur at the nearest available hospital) or for older patients with Medicare. We also did not rebalance our treatment groups during follow-up for time-varying factors such as the use of other medications through methods such as marginal structural models, although the treatment groups were closely balanced on a very extensive set of psychiatric and non-psychiatric medications present at treatment initiation. Given that a very large majority of patients had stopped or modified their initial treatment by 365 days, we did not examine patient outcomes occurring over longer than 365 days in our primary analyses. A follow-up period of 1 year ${ }^{63-65}$ or even briefer ${ }^{66,67}$ is fairly standard for studies examining overall or cause-specific mortality in relation to psychiatric medication initiation, even in cohorts with greater medication adherence. Nevertheless, to the degree that either lithium or valproate is associated with health risks or benefits that accrue over $>1$ year of treatment, the impact of these risks and benefits upon mortality will not be reflected in this study.

As a pilot analysis, we extended the follow-up time we investigated from 0-365 days to 0-1095 days ( 3 years), and found this made minimal difference in the results (intention-to-treat $\mathrm{HR}=0.93,95 \%$ CI $0.87-1.00, P=0.04$, as-initially treated $\mathrm{HR}=$ $0.64,95 \%$ CI $0.48-0.87, P=0.004$ ), although the intent-to-treat findings were now significant at the 0.05 level. Very little of the additional intent-to-treat follow-up time, however, relates to patients still receiving initial treatment, and very little of the as-initially treated follow-up time relates to treatment exposure after the first year of treatment.

Patients with several major mental health diagnoses were included to achieve sufficient power (for example depression, bipolar and psychotic diagnoses). Although the psychiatric diagnoses were each balanced closely between treatments by hdPS-matching, this may have introduced some heterogeneity in the associations between treatments and mortality. Suicide deaths, which some studies have reported as strongly influenced by lithium treatment ${ }^{68,69}$ and/or its discontinuation, ${ }^{70}$ may have been miscoded to some extent as accidents/injuries, resulting in an outcome not completely specific for non-suicide mortality. Studies of overall mortality have also been criticised in general for their lack of specificity. ${ }^{71}$ However, an overall non-suicide mortality focus for this study appears appropriate, given that lithium and valproate affect so many organ systems that a priori cause-specific hypotheses are difficult.

One potential limitation that can affect some non-randomised cohort studies are biases arising from inclusion of patients either in the midst of treatment ('prevalent users') or who have been exposed to either study agent in the past ('past users') (online supplement DS13). We sought to minimise these potential biases through an 'incident first-user design', whereby we only examined patients during their first use of either lithium or valproate after achievement of a 6 month or longer 'clean period' of no exposure. However, a small, extremely similar fraction of patients in each treatment group did have record of past (but not recent exposure) to lithium or valproate ( $12.0 \%$ v. $11.9 \%$, respectively). Excluding these individuals produced little change to the effect estimates (365-day intention-to-treat $\mathrm{HR}=0.89, \quad 95 \%$ CI $0.82-1.03$, $P=0.11 ;$ 365-day as-treated $\mathrm{HR}=0.66,95 \%$ CI $0.46-0.95$, $P=0.02)$. Unfortunately, it is not possible to similarly control for the possibility that some uncertain number of patients may have received one of these medications prior to 1999 (when VHA medication dispensing began to be electronically recorded), or received either medication from outside sources (either recently or in the past). To reduce the concern about recent outside use of lithium or valproate, we only included the patients that we judged were particularly unlikely to have unrecorded outside use by requiring all patients to have more than a year of prior VHA system use. In addition, almost all patients (approximately 95\%) had already been using VHA pharmacy services as well.

The rates of treatment impersistence among patients initiating lithium and valproate were quite high (median time to discontinuation of initial treatment approximately 90 days). Possible reasons for this high rate of treatment impersistence may relate to the high rates of psychiatric comorbidities (for example post-traumatic stress disorder), substance use disorders ${ }^{72}$ and homelessness in our Veteran sample compared with other patient samples. However, the treatment impersistence rates appear to be consistent with those observed in the only other incident cohort from a broad sample of US patients that we were able to identify. Johnson \& McFarland reported a median time to discontinuation of the first episode of treatment with lithium of only 72 days in a US Health Maintenance Organization sample. ${ }^{56}$ In contrast, findings from a non-US incident cohort (a nationwide sample from Denmark) indicated higher rates of persistence with lithium treatment (median time to discontinuation of 181 days). ${ }^{57}$ However, the authors note that their sample may have had less severe mental illness than many cohorts, since $>50 \%$ of the lithium prescriptions were apparently initiated by general practitioners, not psychiatrists. ${ }^{57}$

Although methodological work in comparative effectiveness research has been steadily advancing, it has not been determined whether outcome-focused propensity scores should be favoured over larger propensity scores in all circumstances. Of note, the results given here for the outcome-focused model and in online supplement DS5 for the initial model are generally consistent in many aspects. These aspects include a significant intent-to-treat difference between lithium and valproate at 90 days and substantial effect sizes for as-treated and former users that are almost uniformly consistent in direction of effects, although not always identical in significance. There are a number of reasons, both 
theoretical and specifically from the data itself, that suggest the outcome-focused approach, designed to limit unintended amplification of confounding, is likely less biased and more valid (online supplement DS5). However, because these methods are still being refined, a definite conclusion cannot be reached.

Our study examines a US Veteran sample, so its generalisability to non-Veteran samples is uncertain. (For instance, $86 \%$ of our sample is male. To the extent that the association of lithium or valproate might be modified by a patient's gender, the results here might differ from those observed in patient samples with a more typical gender distribution. Investigating such a possibility, however, will likely require even larger patient samples than examined here). Perhaps less obviously, intent-to-treat estimates (essential for developing a full view of the possible risks and benefits of treatment) produce potential limitations to generalisability. For intent-to-treat estimates to likely generalise to other patient samples, that patient sample would need to also exhibit a similar rate of treatment persistence. If active lithium treatment does have a genuine association with lower mortality risks, however, then cohorts with greater treatment persistence than the patients studied here would generally be expected to show greater benefits. Nevertheless, given that residual confounding appears to bias in the direction of patients being initiated on lithium being at some degree of lower mortality risk, the significant risks observed after lithium discontinuation over 0-180 days (albeit time-limited, in that they are no longer significant over 0-365 days) are actually the mortality findings least likely to be attributable to baseline confounding.

\section{Indirect $v$. direct effects of lithium on mortality}

Whether any non-suicide mortality differences between lithium and valproate are primarily as a result of their direct psychiatric effects (stabilising mood and/or destabilising mood on discontinuation), indirect effects on physical health (for example mood stability possibly leading to better adherence to medical treatment) or direct effects on physical health (both medications affect many organ systems) remains to be elucidated. The possibility of associations between lithium treatment and mortality operating through lithium's effect on psychiatric status might unify the observations of decreased risks associated with active treatment and increased risks associated with discontinuation, given lithium's established impact upon mood in both circumstances. Of note, some recent randomised ${ }^{73}$ and non-randomised ${ }^{74}$ studies have suggested lithium may have greater efficacy in bipolar disorder than valproate. In the BALANCE trial, the valproate treatment arm underperformed both the lithium-valproate combination and lithium alone treatment arms. ${ }^{73}$ In Denmark, lithium was found to be associated with fewer subsequent admissions to psychiatric hospital than valproate. ${ }^{74}$

\section{Implications}

This cohort study of US VHA patients observed significantly reduced non-suicide mortality among all patients initiated on lithium compared with valproate over 0-90 days but not beyond this period. Furthermore, significant associations were observed in opposite directions in secondary analyses: reduced mortality associated with individuals receiving lithium treatment, and increased mortality associated with individuals discontinuing lithium treatment (over 0-180 days), relative to valproate. This pattern suggests a potential dual nature to the mortality risk associations observed between lithium and valproate: mortality benefits associated with active lithium treatment that potentially exceed or are exceeded by counterbalancing risks associated with lithium discontinuation. Intrinsic uncertainties common to non-randomised studies (for example confounding), despite our efforts to minimise them, preclude a definitive judgement of whether lithium, compared with valproate, initiation was associated with net mortality benefit or net harm. One clear and important clinical conclusion nevertheless emerges: once lithium treatment has been initiated, patients and providers should strive to maximise persistence with lithium treatment when feasible and clinically indicated. Such a conclusion results regardless of whether lithium is associated with benefits during active treatment or harms after discontinuation. Given the problem of premature mortality in patients with serious mental illness, ${ }^{21-24}$ the potential mortality differences between lithium and valproate should immediately become a greater research and clinical focus.

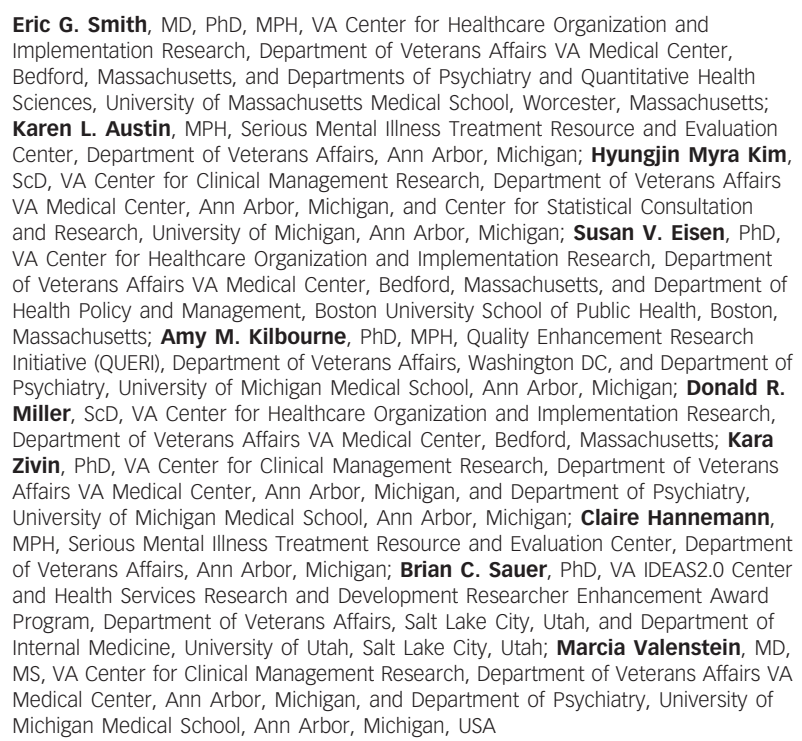

Correspondence: Dr Eric G. Smith, Edith Nourse Rogers Memorial Veterans Hospital, 200 Springs Road, Bedford, MA 01730, USA. Email: eric.smith5@va.gov

First received 14 Sep 2013, final revision 5 Aug 2014, accepted 26 Sep 2014

\section{Funding}

This work was financially supported by a Health Services Research and Development Service (HSR\&D) Career Development Award (CDA-09-216; E.G.S), by funding from the VHA HSRD Center for Healthcare Organization and Implementation Research and by technical and programming support from the VHA Serious Mental Illness Treatment, Resource, and Evaluation Center, Ann Arbor, Michigan. Databases were constructed in part through funding from VHA HSR\&D MRP 03-320 and the VHA Serious Mental IIIness Treatment, Resource, and Evaluation Center. The sponsor had no role in the design and conduct of the study; collection, management, analysis and interpretation of the data; and preparation, review or approval of the manuscript. The views expressed in this article are those of the authors and do not necessarily reflect the position or policy of the Department of Veterans Affairs or the United States government.

\section{Acknowledgements}

We would like to thank two anonymous reviewers whose comments significantly improved this manuscript. The work reported here served as partial fulfilment of the requirements for E.G.S.'s PhD thesis.

\section{References}

1 Morton WA, Sonne SC, Lydiard RB. Lithium side effects in the medically ill. Int J Psychiatry Med 1993; 23: 357-82.

2 Frost RE, Messiha FS. Clinical uses of lithium salts. Brain Res Bull 1983; 11: 219-31.

3 Carmen J, Okafor K, Ike E. The effects of lithium therapy on leukocytes: a 1-year follow-up study. J Natl Med Assoc 1993; 85: 301-3.

4 Colton CW, Manderscheid RW. Congruencies in increased mortality rates, years of potential life lost, and causes of death among public mental health clients in eight states. Prev Chronic Dis 2006; 3: A42. 
5 Manji HK, Moore GJ, Chen G. Clinical and preclinical evidence for the neurotrophic effects of mood stabilizers: implications for the pathophysiology and treatment of manic-depressive illness. Biol Psychiatry 2000; 48: 740-54

6 Hicks D. Lithium induced renal toxicity-a review of the literature. S D J Med 1991; 44: 343-5

7 Gitlin M. Lithium and the kidney: an updated review. Drug Saf 1999; 20: 231-43.

8 Bocchetta A, Loviselli A. Lithium treatment and thyroid abnormalities. Clin Pract Epidemol Ment Health 2006; 2: 23.

9 Weintraub M, Hes JP, Rotmensch HH, Soferman G, Liron M. Extreme sinus bradycardia associated with lithium therapy. ISr J Med Sci 1983; 19 353-5.

10 Montalescot G, Levy Y, Hatt PY. Serious sinus node dysfunction caused by therapeutic doses of lithium. Int J Cardiol 1984; 5: 94-6.

11 Wolf ME, Ranade V, Molnar J, Somberg J, Mosnaim AD. Hypercalcemia, arrhythmia, and mood stabilizers. J Clin Psychopharmacol 2000; 20: 260-4.

12 Norton B, Whalley LJ. Mortality of a lithium-treated population. Br J Psychiatry 1984; 145: 277-82.

13 Coppen A, Standish-Barry H, Bailey J, Houston G, Silcocks P, Hermon C. Does lithium reduce the mortality of recurrent mood disorders? J Affect Disord 1991; 23: 1-7.

14 Vestergaard $\mathrm{P}$, Aagaard J. Five-year mortality in lithium-treated manic-depressive patients. J Affect Disord 1991; 21: 33-8.

15 Muller-Oerlinghausen $B$, Ahrens $B$, Grof E, Grof $P$, Lenz G, Schou M, et al. The effect of long-term lithium treatment on the mortality of patients with manic-depressive and schizoaffective illness. Acta Psychiatr Scand 1992; 86: 218-22.

16 Ahrens B, Muller-Oerlinghausen B, Schou M, Wolf T, Alda M, Grof E, et al. Excess cardiovascular and suicide mortality of affective disorders may be reduced by lithium prophylaxis. J Affect Disord 1995; 33: 67-75.

17 Nilsson A. Mortality in recurrent mood disorders during periods on and of lithium. A complete population study in 362 patients. Pharmacopsychiatry 1995; 28: 8-13.

18 Brodersen A, Licht RW, Vestergaard P, Olesen AV, Mortensen PB. Sixteen-year mortality in patients with affective disorder commenced on lithium. Br J Psychiatry 2000; 176: 429-33.

19 Angst $\mathrm{F}$, Stassen $\mathrm{HH}$, Clayton PJ, Angst J. Mortality of patients with mood disorders: follow-up over 34-38 years. J Affect Disord 2002; 68: 167-81.

20 Cipriani A, Pretty $\mathrm{H}$, Hawton $\mathrm{K}$, Geddes JR. Lithium in the prevention of suicidal behavior and all-cause mortality in patients with mood disorders: a systematic review of randomized trials. Am J Psychiatry 2005; 162: 1805-19.

21 Kilbourne AM, Morden NE, Austin K, Ilgen M, McCarthy JF, Dalack G, et al. Excess heart-disease-related mortality in a national study of patients with mental disorders: identifying modifiable risk factors. Gen Hosp Psychiatry 2009; 31: 555-63.

22 Harris EC, Barraclough B. Excess mortality of mental disorder. Br J Psychiatry 1998: 173: $11-53$

23 Miller BJ, Paschall CB, 3rd, Svendsen DP. Mortality and medical comorbidity among patients with serious mental illness. Psychiatr Serv 2006; 57: 1482-7.

24 Zivin K, Ilgen MA, Pfeiffer PN, Welsh DE, McCarthy J, Valenstein M, et al. Early mortality and years of potential life lost among veterans affairs patients with depression. Psychiatr Serv 2012; 63: 823-6.

25 Young $\mathrm{AH}$, Hammond $\mathrm{JM}$. Lithium in mood disorders: increasing evidence base, declining use? Br J Psychiatry 2007; 191: 474-6.

26 Blanco $\mathrm{C}$, Laje G, Olfson M, Marcus SC, Pincus HA. Trends in the treatmen of bipolar disorder by outpatient psychiatrists. Am J Psychiatry 2002; 159: 1005-10.

27 Wolfsperger M, Greil W, Rossler W, Grohmann R. Pharmacological treatment of acute mania in psychiatric in-patients between 1994 and 2004. J Affect Disord 2007; 99: 9-17.

28 Shulman $\mathrm{KI}$, Rochon $\mathrm{P}$, Sykora $\mathrm{K}$, Anderson G, Mamdani M, Bronskill S, et al. Changing prescription patterns for lithium and valproic acid in old age: shifting practice without evidence. BMJ 2003; 326: 960-1.

29 Blow FC, Valenstein M, Austin K, Khanuja K, McCarthy JF. Specialty Care for Veterans with Depression in the VHA: 2002 National Depression Registry Report. VA National Serious Mental Illness Treatment Research \& Evaluation Center (SMITREC), VHA Health Services Research \& Development, 2003.

30 Ray WA. Evaluating medication effects outside of clinical trials: new-user designs. Am J Epidemiol 2003; 158: 915-20.
31 Hernan MA, Robins JM. Authors' response, part I: observational studies analyzed like randomized experiments: best of both worlds. Epidemiology 2008; 19: 789-92.

32 Cooper WO, Habel LA, Sox CM, Chan KA, Arbogast PG, Cheetham TC, et al. ADHD drugs and serious cardiovascular events in children and young adults. N Engl J Med 2011; 365: 1896-904.

33 Cowper DC, Kubal JD, Maynard C, Hynes DM. A primer and comparative review of major US mortality databases. Ann Epidemiol 2002; 12: 462-8.

34 Schneeweiss S, Rassen JA, Glynn RJ, Avorn J, Mogun H, Brookhart MA High-dimensional propensity score adjustment in studies of treatment effects using health care claims data. Epidemiology 2009; 20: 512-22.

35 Patorno E, Bohn RL, Wahl PM, Avorn J, Patrick AR, Liu J, et al. Anticonvulsant medications and the risk of suicide, attempted suicide, or violent death. JAMA 2010; 303: 1401-9.

36 Wyss R, Girman CJ, Locasale RJ, Alan Brookhart M, Sturmer T. Variable selection for propensity score models when estimating treatment effects on multiple outcomes: a simulation study. Pharmacoepidemiol Drug Saf 2013; 22: 77-85.

37 Selim AJ, Berlowitz DR, Fincke G, Rosen AK, Ren XS, Christiansen CL, et al. Risk-adjusted mortality rates as a potential outcome indicator for outpatient quality assessments. Med Care 2002; 40: 237-45.

38 Brookhart MA, Schneeweiss S, Rothman KJ, Glynn RJ, Avorn J, Sturmer T. Variable selection for propensity score models. Am J Epidemiol 2006; 163 1149-56.

39 Patrick AR, Schneeweiss S, Brookhart MA, Glynn RJ, Rothman KJ, Avorn J, et al. The implications of propensity score variable selection strategies in pharmacoepidemiology: an empirical illustration. Pharmacoepidemiol Drug Saf 2011; 20: 551-9.

40 Pearl J. Invited commentary: understanding bias amplification. Am J Epidemiol 2011; 174: 1223-7.

41 Brooks JM, Ohsfeldt RL. Squeezing the balloon: propensity scores and unmeasured covariate balance. Health Serv Res 2013; 48: 1487-507.

42 Austin PC. Some methods of propensity-score matching had superior performance to others: results of an empirical investigation and Monte Carlo simulations. Biom J 2009; 51: 171-84.

43 Faries DE, Leon AC, Haro JM, Obenchain RL, SAS Institute. Analysis of Observational Health Care Data using SAS. SAS Institute, 2010.

44 Austin PC, Grootendorst P, Anderson GM. A comparison of the ability of different propensity score models to balance measured variables between treated and untreated subjects: a Monte Carlo study. Stat Med 2007; 26: 734-53.

45 Muller-Oerlinghausen B, Wolf $T$, Ahrens B, Glaenz T, Schou M, Grof E, et al. Mortality of patients who dropped out from regular lithium prophylaxis: a collaborative study by the International Group for the Study of Lithium-treated patients (IGSLI). Acta Psychiatr Scand 1996; 94 344-7.

46 Bocchetta A. Mortality follow-up of patients since commencing lithium therapy. J Clin Psychopharmacol 2005; 25: 197-9.

47 Christodoulou GN, Lykouras EP. Abrupt lithium discontinuation in manic-depressive patients. Acta Psychiatr Scand 1982; 65: 310-4.

48 Suppes T, Baldessarini RJ, Faedda GL, Tohen M. Risk of recurrence following discontinuation of lithium treatment in bipolar disorder. Arch Gen Psychiatry 1991; 48: 1082-8.

49 Faedda GL, Tondo L, Baldessarini RJ, Suppes T, Tohen M. Outcome after rapid vs gradual discontinuation of lithium treatment in bipolar disorders. Arch Gen Psychiatry 1993; 50: 448-55

50 Goodwin GM. Recurrence of mania after lithium withdrawal. Implications for the use of lithium in the treatment of bipolar affective disorder. Br J Psychiatry 1994; 164: 149-52.

51 Scott J, Pope M. Nonadherence with mood stabilizers: prevalence and predictors. J Clin Psychiatry 2002; 63: 384-90.

52 Svarstad BL, Shireman TI, Sweeney JK. Using drug claims data to assess the relationship of medication adherence with hospitalization and costs. Psychiatr Serv 2001; 52: 805-11.

53 Schumann C, Lenz G, Berghofer A, Muller-Oerlinghausen B. Non-adherence with long-term prophylaxis: a 6-year naturalistic follow-up study of affectively ill patients. Psychiatry Res 1999; 89: 247-57.

54 Maarbjerg K, Aagaard J, Vestergaard P. Adherence to lithium prophylaxis: I. Clinical predictors and patient's reasons for nonadherence. Pharmacopsychiatry 1988; 21: 121-5.

55 Aagaard J, Vestergaard P. Predictors of outcome in prophylactic lithium treatment: a 2-year prospective study. J Affect Disord 1990; 18: 259-66.

56 Johnson RE, McFarland $\mathrm{BH}$. Lithium use and discontinuation in a health maintenance organization. Am J Psychiatry 1996; 153: 993-1000. 
57 Kessing LV, Sondergard L, Kvist K, Andersen PK. Adherence to lithium in naturalistic settings: results from a nationwide pharmacoepidemiological study. Bipolar Disord 2007; 9: 730-6.

58 Lolich M, Vazquez GH, Alvarez LM, Tamayo JM. Psychosocial interventions in bipolar disorder: a review. Actas Esp Psiquiatr 2012; 40: 84-92.

59 Colom $F$, Vieta E, Sanchez-Moreno J, Palomino-Otiniano R, Reinares $M$ Goikolea JM, et al. Group psychoeducation for stabilised bipolar disorders: 5-year outcome of a randomised clinical trial. Br J Psychiatry 2009; 194 260-5.

60 Kessing LV, Hansen HV, Hvenegaard A, Christensen EM, Dam H, Gluud C et al. Treatment in a specialised out-patient mood disorder clinic $v$. standard out-patient treatment in the early course of bipolar disorder: randomised clinical trial. Br J Psychiatry 2013; 202: 212-9.

61 Goodwin GM. Evidence-based guidelines for treating bipolar disorder: revised second edition-recommendations from the British Association for Psychopharmacology. J Psychopharmacol (Oxf) 2009; 23: 346-88.

62 Chao SH, Urbano FL. Introduction: Medicare Section 1013 and AHRQ's Effective Health Care Program. J Manag Care Pharm 2007; 13 (suppl 1): S3-6.

63 Kales HC, Valenstein M, Kim HM, McCarthy JF, Ganoczy D, Cunningham F, et al. Mortality risk in patients with dementia treated with antipsychotics versus other psychiatric medications. Am J Psychiatry 2007; 164: 1568-76.

64 Schneeweiss S, Patrick AR, Solomon DH, Mehta J, Dormuth C, Miller M, et al. Variation in the risk of suicide attempts and completed suicides by antidepressant agent in adults: a propensity score-adjusted analysis of 9 years' data. Arch Gen Psychiatry 2010; 67: 497-506.

65 Soyka M, Apelt SM, Lieb M, Wittchen HU. One-year mortality rates of patients receiving methadone and buprenorphine maintenance therapy: a nationally representative cohort study in 2694 patients. J Clin Psychopharmacol 2006; 26: $657-60$
66 Wang PS, Schneeweiss S, Avorn J, Fischer MA, Mogun H, Solomon DH, et al. Risk of death in elderly users of conventional vs. atypical antipsychotic medications. N Engl J Med 2005; 353: 2335-41.

67 Kales HC, Kim HM, Zivin K, Valenstein M, Seyfried LS, Chiang C, et al. Risk of mortality among individual antipsychotics in patients with dementia. Am J Psychiatry 2012; 169: 71-9.

68 Baldessarini RJ, Tondo L, Davis P, Pompili M, Goodwin FK, Hennen J. Decreased risk of suicides and attempts during long-term lithium treatment: a meta-analytic review. Bipolar Disord 2006; 8: 625-39.

69 Goodwin FK, Fireman B, Simon GE, Hunkeler EM, Lee J, Revicki D. Suicide risk in bipolar disorder during treatment with lithium and divalproex. JAMA 2003; 290: $1467-73$.

70 Baldessarini RJ, Tondo L, Hennen J. Effects of lithium treatment and its discontinuation on suicidal behavior in bipolar manic-depressive disorders. J Clin Psychiatry 1999; 60 (suppl 2): 77-84.

71 Ray WA. Observational studies of drugs and mortality. N Engl J Med 2005 353: $2319-21$.

72 Manwani SG, Szilagyi KA, Zablotsky B, Hennen J, Griffin ML, Weiss RD. Adherence to pharmacotherapy in bipolar disorder patients with and without co-occurring substance use disorders. J Clin Psychiatry 2007; 68: 1172-6.

73 Geddes JR, Goodwin GM, Rendell J, Azorin JM, Cipriani A, Ostacher MJ, et al. Lithium plus valproate combination therapy versus monotherapy for relapse prevention in bipolar I disorder (BALANCE): a randomised open-label trial. Lancet 2010; 375: 385-95.

74 Kessing LV, Hellmund G, Geddes JR, Goodwin GM, Andersen PK. Valproate v. lithium in the treatment of bipolar disorder in clinical practice: observational nationwide register-based cohort study. Br J Psychiatry 2011; 199: 57-63. 